\title{
Violaxanthin de-epoxidase disulphides and their role in activity and thermal stability
}

\author{
Erik Ingmar Hallin ${ }^{1} \cdot$ Kuo Guo $^{1} \cdot$ Hans-Erik Åkerlund ${ }^{1}$
}

Received: 16 January 2015 / Accepted: 5 March 2015/Published online: 13 March 2015

(c) The Author(s) 2015. This article is published with open access at Springerlink.com

\begin{abstract}
Violaxanthin de-epoxidase (VDE) catalyses the conversion of violaxanthin to zeaxanthin at the lumen side of the thylakoids during exposure to intense light. VDE consists of a cysteine-rich N-terminal domain, a lipocalinlike domain and a negatively charged C-terminal domain. That the cysteines are important for the activity of VDE is well known, but in what way is less understood. In this study, wild-type spinach VDE was expressed in E. coli as inclusion bodies, refolded and purified to give a highly active and homogenous preparation. The metal content ( $\mathrm{Fe}$, $\mathrm{Cu}, \mathrm{Ni}, \mathrm{Mn}, \mathrm{Co}$ and $\mathrm{Zn}$ ) was lower than $1 \mathrm{~mol} \%$ excluding a metal-binding function of the cysteines. To investigate which of the 13 cysteines that could be important for the function of VDE, we constructed mutants where the cysteines were replaced by serines, one by one. For 12 out of 13 mutants the activity dropped by more than $99.9 \%$. A quantification of free cysteines showed that only the most $\mathrm{N}$-terminal of these cysteines was in reduced form in the native VDE. A disulphide pattern in VDE of C9-C27, C14-C21, C33-C50, C37-C46, C65-C72 and C118-C284 was obtained after digestion of VDE with thermolysin followed by mass spectroscopy analysis of reduced versus non-reduced samples. The residual activity found for the mutants showed a variation that was consistent with the results obtained from mass spectroscopy. Reduction of the disulphides resulted in loss of a rigid structure and a decrease in thermal stability of $15^{\circ} \mathrm{C}$.
\end{abstract}

Hans-Erik Åkerlund

hans-erik.akerlund@biochemistry.lu.se

1 Department of Biochemistry and Structural Biology, Lund University, P.O. Box 124, 22100 Lund, Sweden
Keywords Violaxanthin de-epoxidase $\cdot$ Cysteine . Mutation · Disulphide bond · Violaxanthin · Zeaxanthin

\section{Introduction}

Plants and algae need light to drive the photosynthetic machinery. However, an excess of light will cause oxidation damage. To protect against the surplus of light, the energy is converted to heat, in a process called non-photochemical quenching (NPQ). One prerequisite for NPQ is the presence of zeaxanthin/antheraxanthin formed from violaxanthin in the xanthophyll cycle (Demmig-Adams 1990). This conversion is catalysed by violaxanthin de-epoxidase (VDE), located at the lumen side of the thylakoids. When exposed to intense light, the inside of the thylakoids will become acidic, which activates VDE and the conversion from violaxanthin to zeaxanthin. For a review on the xanthophyll cycle background see (Eskling et al. 1997). VDE consists of three domains, a cysteine-rich $\mathrm{N}$-terminal domain with 11 of the total 13 cysteines, a lipocalin-like domain, predicted to bind violaxanthin (Saga et al. 2010) and a glutamate-rich C-terminal domain. The C-terminal is highly variable between different species and has been partially truncated without major loss of activity (Hieber et al. 2002). The three-dimensional structure of a dimer of the lipocalin domain has been determined (Arnoux et al. 2009) and binding of the substrates, violaxanthin and ascorbic acid, to this dimer has been suggested from in silico experiments (Saga et al. 2010). However, the N-terminally truncated protein used for structure determination had no enzymatic activity. Thus, the role of the cysteine-rich $\mathrm{N}$-terminal remains to be established. The cysteines are conserved throughout all known VDE sequences with the exception of the first cysteine (Cys 7), which is not present in algae VDE. This wide conservation 
among different organisms is an indication of the cysteine's importance for the function of VDE. An isolated Arabidopsis thaliana mutant (npq1, Niyogi et al. 1998), unable to convert violaxanthin to zeaxanthin, have exchanged one of these cysteines (Cys 72) for a tyrosine. The inhibition of VDE by dithiothreitol (DTT) suggests that disulphide bonds are necessary for the catalysis (Yamamoto and Kamite 1972). Cysteines in general are also known to coordinate metal ions, participate in catalysis, act as redox sensors or provide structural stability in the form of disulphides.

In this study, we have investigated the role of the cysteines in VDE by mutating all of the cysteines to serines, one by one, which resulted in the loss of activity of all cysteine mutants except for the first cysteine in the sequence (Cys 7). Metal analysis of purified active VDE did not reveal any metals that could potentially be bound to these cysteines. Quantification of free cysteines indicated only one free cysteine per VDE molecule, which suggests that the rest of the 12 cysteines form disulphides. The disulphide pattern was analysed with mass spectroscopy and revealed five out of six possible disulphide bonds. These bonds could give valuable information about the structure of the N-terminal domain. The thermal stability of VDE was reduced upon reduction with DTT, which indicates that one function of the disulphides is to maintain a rigid structure.

\section{Materials and methods}

\section{Gene and original plasmid source}

The original isolation of the spinach VDE gene sequence is described in (Emanuelsson et al. 2003). The mature native sequence of spinach VDE (VDALK...IRKLR) was inserted in $\mathrm{pET} 22 \mathrm{~b}+$ (Novagen) between the restriction sites $N d e 1$ and BamH1 yielding a non-tagged protein with an initial methionine.

\section{Mutation protocol}

The 13 cysteine mutants, where one cysteine per mutant have been exchanged to a serine, were constructed using the overlap extension polymerase chain reaction (Ho et al. 1989) with primers, shown in supplemental data, on pET22b+ containing the native sequence of spinach VDE. The amplicon of each mutant was inserted in pET22b+ between the restriction sites $N d e 1$ and Bam $\mathrm{H} 1$.

\section{Protein isolation and purification}

Overexpression of recombinant VDE was done in Escherichia coli BL21(DE3) grown in lysogeny broth at $37^{\circ} \mathrm{C}$, induced with isopropyl $\beta$-D-1-thiogalactopyranoside (1 mM) at $\mathrm{OD}_{600} \approx 0.6$ for $2 \mathrm{~h}$ at $37^{\circ} \mathrm{C}$. The cells were harvested by centrifugation, washed with Tris-HCl (100 mM, pH 8.0), $\mathrm{NaCl}(170 \mathrm{mM})$ and disrupted by French press in Tris- $\mathrm{HCl}$ (20 mM, pH 8.0), EDTA (10 mM), DTT (5 mM) and Triton $\mathrm{X}-100(1 \%, \mathrm{v} / \mathrm{v})$. Inclusion bodies were isolated by centrifugation, washed three times with Tris- $\mathrm{HCl}(20 \mathrm{mM}, \mathrm{pH}$ 8.0), EDTA (10 mM), DTT (5 mM), Triton X-100 (1\%, $\mathrm{v} / \mathrm{v}$ ) and once without Triton X-100. The washed inclusion bodies were dissolved in urea $(8 \mathrm{M})$, Tris- $\mathrm{HCl}(20 \mathrm{mM}, \mathrm{pH}$ 8.0), EDTA (10 mM), DTT (5 mM) and centrifuged to remove insoluble material. The refolding of the solubilised inclusion bodies were done by removing urea with a PD-10 gel filtration column, followed by incubation for $60 \mathrm{~min}$ and another PD-10 step to remove DTT. Reduced glutathione $(2 \mathrm{mM})$ and oxidised glutathione $(0.3 \mathrm{mM})$ were added and the mixture was incubated for $16 \mathrm{~h}$ at $22^{\circ} \mathrm{C}$. The refolded protein was purified by size-exclusion chromatography (HiPrep 26/60 Sephacryl S-200) in Tris-HCl (20 mM, pH 8.0), EDTA(10 mM), and $\mathrm{NaCl}(100 \mathrm{mM})$, collecting the monomeric VDE fraction and concentrating the protein using a $10 \mathrm{kDa}$ cut-off centrifugation filter. The protein concentration was determined using the Bradford method (Bradford 1976) and the theoretical absorbance at $280 \mathrm{~nm}$. The VDE activity was measured at $26^{\circ} \mathrm{C}$ in citrate-phosphate buffer (50 mM citrate, $110 \mathrm{mM}$ phosphate, $\mathrm{pH}$ 5.2), violaxanthin ( $0.33 \mu \mathrm{M}$, extracted from spinach leaves according to Thayer and Björkman 1990), monogalactosyldiacylglycerol (9 $\mu \mathrm{M})$ and ascorbate $(30 \mathrm{mM})$ by dual-wavelength measurements (502-540 nm), using a Shimadzu UV-3000 spectrophotometer according to (Yamamoto and Higashi 1978).

\section{Isolation and activity of cysteine mutants}

Induced and washed $E$. coli BL21(DE3) cells (40 mg) with overexpressed native or mutated VDE were resuspended in $1.0 \mathrm{ml}$ Tris- $\mathrm{HCl}$ (85 mM, pH 8.0), $\mathrm{NaCl}$ (85 mM), EDTA (0.85 mM), Triton X-100 (1\%, v/v) and lysozyme (0.5 g/l). After five freeze/thaw cycles, $\mathrm{CaCl}_{2}(10 \mathrm{mM})$ and DNase I $(0.1 \mathrm{~g} / \mathrm{l})$ were added followed by incubation for $30 \mathrm{~min}$, and centrifugation to collect the inclusion bodies. The pellet was washed two times with Tris- $\mathrm{HCl}(90 \mathrm{mM}, \mathrm{pH} 8.0), \mathrm{NaCl}$ (90 mM), EDTA (0.9 mM), Triton X-100 (1\%, v/v) and once without Triton $\mathrm{X}-100$. The inclusion bodies were dissolved with urea $(8 \mathrm{M})$, Tris- $\mathrm{HCl}(60 \mathrm{mM}, \mathrm{pH} 8.0), \mathrm{NaCl}$ $(60 \mathrm{mM})$, EDTA $(0.6 \mathrm{mM})$ and centrifuged to remove insoluble material. The supernatant was dialysed against $50 \mathrm{ml}$ of Tris- $\mathrm{HCl}$ (100 mM, pH 8.0), NaCl (100 mM), EDTA $(1 \mathrm{mM})$ for $16 \mathrm{~h}$ at $22{ }^{\circ} \mathrm{C}$ using $10-14 \mathrm{kDa}$ dialysis tubing (Spectra/Por). The refolded protein was centrifugated at $100,000 \times g$ for $60 \mathrm{~min}$ to remove the aggregated protein.

The VDE activity was measured by mixing refolded VDE with citrate-phosphate buffer $(50 \mathrm{mM}$ citrate, $110 \mathrm{mM}$ phosphate, $\mathrm{pH} 5.2)$, violaxanthin $(8.6 \mu \mathrm{M}$, extracted from 
spinach leaves according to Thayer and Björkman 1990, monogalactosyldiacylglycerol $(24 \mu \mathrm{M})$, ascorbate $(30 \mathrm{mM})$ and incubated at $22^{\circ} \mathrm{C}$ for $270 \mathrm{~min}$. After the reaction, the $\mathrm{pH}$ was raised to seven with $\mathrm{NaOH}$. The xanthophylls were extracted by addition of acetone ( $80 \%$ final concentration) and centrifuged to remove insoluble material. The solvent of the supernatant was evaporated with a vacuum concentrator (Christ, RVC 2-18), to be replaced with ethyl acetate $(100 \%)$ and centrifuged to remove insoluble material, followed by another solvent exchange to methanol (100\%). The concentrations of violaxanthin, antheraxanthin and zeaxanthin were quantified by reversed-phase HPLC (Waters 600E, 996), as described in (Clausén et al. 2010).

\section{Quantification of free cysteines}

The number of thiols in VDE was quantified using 5,5'dithiobis-(2-nitrobenzoic acid) (DTNB), similar to (Sedlak and Lindsay 1968) in Tris- $\mathrm{HCl}(200 \mathrm{mM}, \mathrm{pH}$ 8.5), EDTA $(1.0 \mathrm{mM})$, SDS $(0.5 \% \mathrm{w} / \mathrm{v})$, DTNB $(0.2 \mathrm{mM})$ and $2.9 \mu \mathrm{M}$ wild-type VDE or C7S mutant, using a Shimadzu UV-Vis spectrophotometer (UV-2101PC) and L-cysteine as calibration standard.

\section{Mass spectroscopy}

Enzymatic digestion of purified VDE $(10 \mu \mathrm{g})$ was performed by incubation with thermolysin $(1 \mu \mathrm{g}$, from Promega) in $60 \mu \mathrm{l}$ Tris- $\mathrm{HCl}(50 \mathrm{mM}, \mathrm{pH} 8.0), \mathrm{CaCl}_{2}(1 \mathrm{mM})$ at $80{ }^{\circ} \mathrm{C}$ for $30 \mathrm{~min}$. For reduction of disulphides $1 \mu \mathrm{l}$ of DTT $(500 \mathrm{mM})$ was added to $10 \mu \mathrm{l}$ of the peptide sample and incubated for $20 \mathrm{~min}$ at $22{ }^{\circ} \mathrm{C}$. Acidification of both reduced and non-reduced samples were done by the addition of trifluoroacetic acid (TFA) to $0.5 \%(\mathrm{v} / \mathrm{v})$. The peptide samples $(0.5 \mu \mathrm{l})$ were spotted on the MALDI-target plate together with $0.5 \mu \mathrm{l}$ of the matrix ( $\alpha$-cyano-4hydroxycinnamic acid $(10 \mathrm{mg} / \mathrm{ml})$ in $70 \%$ acetonitrile, $29 \%$ water, $1 \%$ TFA). Alternatively, the non-reduced peptides (after addition of TFA) were separated by reversephase nano-LC using a 1100 Series Nanoflow LC system (Agilent Technologies, Waldbronn, Germany) with a precolumn (Zorbax $300 \mathrm{SB}$ C18, $5 \times 0.3 \mathrm{~mm}$ ) and separation column (Zorbax $300 \mathrm{SB}$ C18, $150 \times 0.075 \mathrm{~mm}$ ). Peptides were eluted with an acetonitrile gradient (1-90\% acetonitrile in water, with $0.1 \%$ TFA) and collected on a stainless steel MALDI-target using an 1100 Series LC microcollection spotting system. For reduction of separated peptides, TCEP $(0.3 \mu \mathrm{l}, 10 \mathrm{mM})$ was added to the MALDItarget before addition of the matrix $(0.2 \mu \mathrm{l}$, same concentrations as above). MS and MS/MS spectra were collected using a 4700 MALDI TOF/TOF mass spectrometer (Applied Biosystems, Framingham, CA, USA) in positive reflector mode.

\section{Metal analysis}

The concentration of $\mathrm{Fe}, \mathrm{Cu}, \mathrm{Ni}, \mathrm{Mn}, \mathrm{Co}$ and $\mathrm{Zn}$ was measured by inductively coupled plasma mass spectrometry (ICP-MS) for purified VDE $(14 \mu \mathrm{M})$, with added nitric acid (1\%), using an Aurora Elite (Bruker).

\section{Thermal stability measurement with dynamic light scattering}

The thermal stability was measured for purified VDE $(18 \mu \mathrm{M})$ in Tris- $\mathrm{HCl}(60 \mathrm{mM}, \mathrm{pH} 8.0), \mathrm{NaCl}(100 \mathrm{mM})$ and EDTA (5.5 mM), using a Zetasizer Nano $\mathrm{S}$ (Malvern Instruments, Malvern, UK). The reduced VDE sample also contained DTT $(10 \mathrm{mM})$. The z-average cumulant size (ISO 13321 1996) was recorded at $3{ }^{\circ} \mathrm{C}$ intervals from 15 to $70{ }^{\circ} \mathrm{C}$ with $60 \mathrm{~s}$ of equilibration time.

\section{Results}

\section{Protein characteristics}

The recombinant spinach VDE expressed in E. coli as inclusion bodies, refolded and purified as a monomeric protein was $>98 \%$ pure based on SDS-PAGE and Coomassie staining (not shown). The enzyme was highly active and showed a specific activity of $208 \mu \mathrm{kat} / \mathrm{g}$ protein $(750 \mu \mathrm{mol} /$ $\mathrm{mg} / \mathrm{h}$ ), which is similar to the value for native VDE purified from spinach (Arvidsson et al. 1996). The final yield of enzyme was $2 \mathrm{mg} / \mathrm{l}$ of growth medium. The recombinant protein did not show any obvious prosthetic group, since no absorbance could be detected in the visible range of the spectra even at concentrations as high as $10 \mathrm{mg} / \mathrm{ml}$ (not shown). Some prosthetic groups may involve metal ions bound to cysteine ligands without showing any absorbance. To check this possibility, metals that potentially could be bound to cysteines were analysed in the purified recombinant and active VDE. The metals $\mathrm{Fe}, \mathrm{Cu}, \mathrm{Ni}, \mathrm{Mn}, \mathrm{Co}$ and $\mathrm{Zn}$ were all found in amounts less than $1 \%$ of VDE on a molar basis and are therefore neither not likely to be bound to the enzyme nor required for the enzymatic activity.

\section{Violaxanthin de-epoxidase activity of the cysteine mutants}

The mutation of a cysteine to a serine resulted in an activity loss (Fig. 1) of more than $99.9 \%$ for all cysteine mutants except for C7S. Thus all cysteines, except C7, are important for the enzyme function, and are consistent with the conservation of these cysteines throughout evolution. The low activity of $\mathrm{C} 72 \mathrm{~S}$ mutant, corresponding to the cysteine mutated in $n p q 1$, is coherent with the incapability of $n p q 1$ to 
Fig. 1 The specific activity of the cysteine mutants, normalised to the specific activity of native spinach VDE (WT). A magnification of the mutants with low activity is shown in a window to visualise the difference in remaining activity

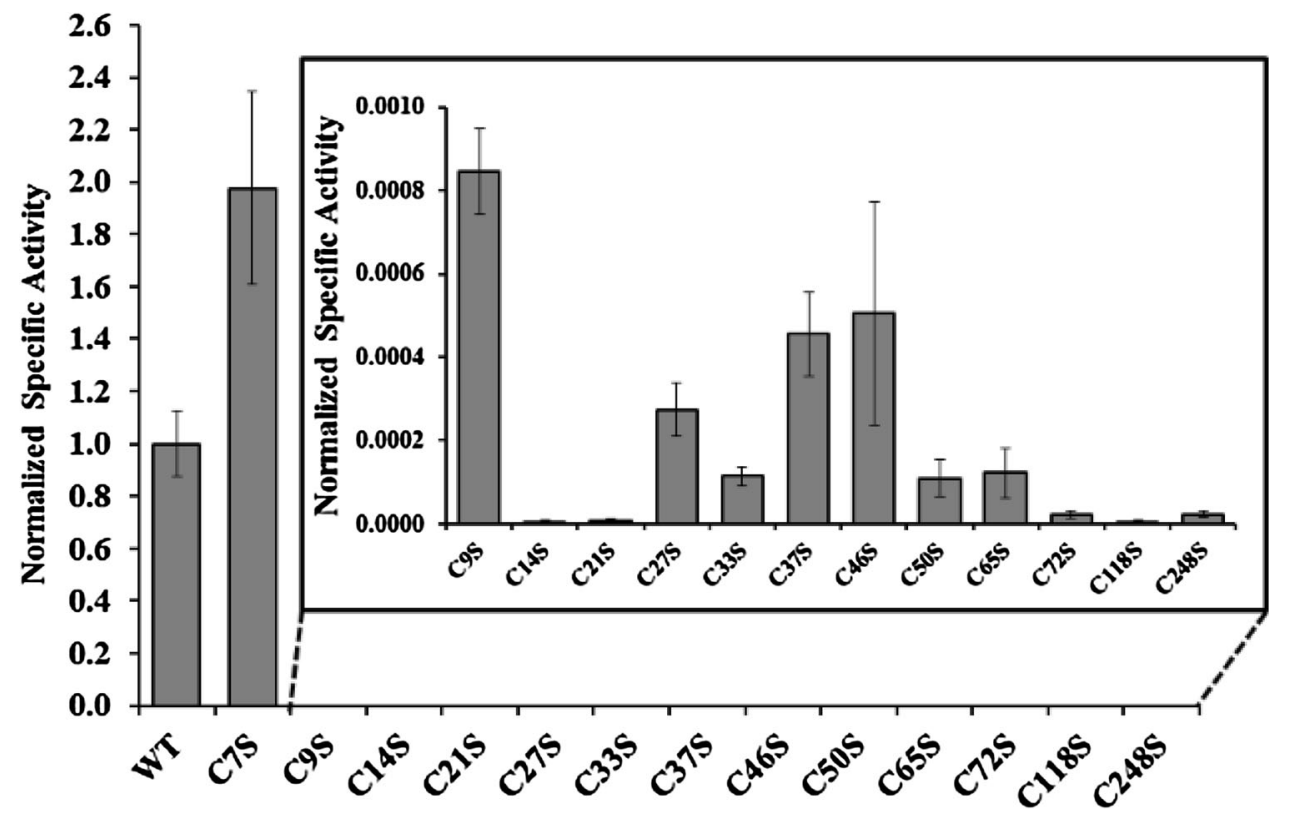

accumulate zeaxanthin (Niyogi et al. 1998). Even though most of the mutants lost almost all activity, all of them still were catalytically active and produced both antheraxanthin and zeaxanthin. As the degree of conversion of violaxanthin was very low under the conditions used here, the dominating product was antheraxanthin, but zeaxanthin could also be clearly detected (not shown). A very low activity from a potentially inactive mutant could be possible due to translational misreading errors, which has been estimated to occur in the range of $10^{-3}-10^{-4}$ per codon (Kramer and Farabaugh 2007). Thus, a low amount of active VDE with the wild-type sequence could be present while most of the enzyme molecules were completely inactive, rather than that each enzyme molecule shows a low activity. However, the activities showed large differences depending on which cysteine was mutated, with less than $0.0005 \%$ activity remaining for C14S and more than 100-fold higher activity remaining for the C9S mutant. The C7S mutant was completely different. It was not inhibited at all but showed even higher specific activity than the wild-type VDE. Thus, this cysteine is not important for the enzymatic function, under the assay conditions tested, and can be replaced by a serine. This could explain why Cys 7 is not conserved in algae VDE.

A basic question is if the cysteines in VDE are in the oxidised form as disulphides or in the reduced form as free cysteine groups. The quantification of free cysteines in the recombinant wild-type VDE gave a molar ratio of $0.87 \pm 0.24$ thiols per VDE molecule which indicates that wild-type VDE has only one free cysteine. In contrast, the C7S mutant gave a molar ratio of $0.28 \pm 0.10$ thiols per VDE. This value is closer to zero than two and indicates that Cys 7 is the free and reduced cysteine in wild-type
VDE. All the other cysteines should be in the form of disulphides, but which cysteines are coupled to each other?

\section{Disulphide pattern}

Thermolytic digestion of purified recombinant VDE without reduction of the cysteines will maintain the native disulphide bonds. To identify peptides containing a disulphide bond, reduced and non-reduced samples were compared. A mass peak, only found in the non-reduced sample and not in the reduced sample is an indication of a peptide containing a disulphide bond. If the disulphide bond was an internal linkage within the same peptide, the reduction will cause a mass shift of $+2 \mathrm{Da}$. A disulphide bond between two peptides will, upon reduction, show two new mass peaks in the reduced sample. The mass of the two linked peptides will hint possible disulphide connections by combining the mass of other cysteinecontaining peptides. Peptides containing a disulphide bond were confirmed by MS/MS, when at least three well-defined peaks were found supporting the prediction.

By analysing the mass of peptides containing a disulphide bond, five out of six possible disulphide bonds could be identified (Table 1 and Fig. 2). No peptides with alternative disulphide connections were found. One of the disulphide bonds found, connects Cys 118 and Cys 248. This pair is located inside the lipocalin domain and the result is in agreement with the X-ray structure of the lipocalin domain of the recombinant VDE of Arabidopsis thaliana (Arnoux et al. 2009). No peptide representing Cys 7 , Cys 9 or Cys 27 could be positively identified. If these cysteines form disulphides or not could therefore not be confirmed with mass spectroscopy. However, the results 
Table 1 List of peptides containing a disulphide bond found using mass spectroscopy and confirmed with MS/MS

\begin{tabular}{llllll}
\hline $\begin{array}{l}\text { Disulphide } \\
\text { bond }\end{array}$ & $\begin{array}{l}\text { Observed } \\
\text { mass (Da) }\end{array}$ & $\begin{array}{l}\text { Theoretical } \\
\text { mass (Da) }\end{array}$ & $\begin{array}{l}\text { Observed } \\
\text { mass after } \\
\text { reduction (Da) }\end{array}$ & $\begin{array}{l}\text { Theoretical mass } \\
\text { after reduction (Da) }\end{array}$ & Sequence \\
\hline $14-21$ & 1303.67 & 1303.69 & 1305.69 & 1305.70 & LKECRIELAKC \\
$14-21$ & 1416.76 & 1416.77 & 1418.79 & 1418.79 & LLKECRIELAKC \\
$14-21$ & 1434.77 & 1434.78 & - & - & LLKECRIE+AKCI \\
$37-46$ & 1648.67 & 1648.67 & 1650.67 & 1650.68 & LQTCNNRPDETECQ \\
$118-248$ & 1808.76 & 1808.76 & - & - & FDCQ+IRTDNTCGPEPP \\
$118-248$ & 2521.09 & 2521.08 & - & - & FDAFDCQLHE+IRTDNTCGPEPP \\
$118-248$ & 2567.14 & 2567.12 & - & - & LNPTFDAFDCQ+IRTDNTCGPEPP \\
$33-37-46-50$ & 2696.17 & 2696.16 & 2700.19 & 2700.19 & VACLQTCNNRPDETECQIKCGDLF \\
$118-248$ & 2714.21 & 2714.19 & - & - & LNPTFDAFDCQ+FIRTDNTCGPEPP \\
$37-46-50$ & 2738.25 & 2738.23 & 2740.25 & 2740.25 & LQTCNNRPDETECQIKCGDLFANK \\
$118-248$ & 3018.47 & 3018.37 & - & - & IRTDNTCGPEPPLVER+FDAFDCQLHE \\
$118-248$ & 3165.56 & 3165.44 & - & 3405.65 & FIRTDNTCGPEPPLVER+FDAFDCQLHE \\
$65-72$ & 3403.77 & 3403.63 & 3405.65 & 3931.99 & VVDEFNECAVSRKKCVPQKSDVGEFPVPDPS \\
$65-72$ & 3930.10 & 3929.98 & 3932.04 & VVDEFNECAVSRKKCVPQKSDVGEFPVPDPSVLVKS
\end{tabular}

The mass of peptides with intramolecular disulphides after reduction are also shown. Intramolecular disulphides are shifted $+2 \mathrm{Da}$ after reduction, while reduction of intermolecular disulphides splits the linked peptides into two

presented in the last section showed that it was only the Cys 7 that was in the reduced form, and all the other cysteines would be engaged in disulphide linkages. This suggests indirectly that the two remaining cysteines, Cys 9 and Cys 27, form one disulphide linkage with each other (dotted line in Fig. 2). As one possible role for the disulphides would be to stabilise the structure of VDE, we studied the heat denaturation of the non-reduced and reduced protein by dynamic light scattering.

\section{Thermal stability of reduced and non-reduced VDE}

The apparent melting temperature for the non-reduced VDE was found at around $48{ }^{\circ} \mathrm{C}$ (Fig. 3). After reduction with DTT, the melting temperature was lowered by $15^{\circ} \mathrm{C}$, to around $33{ }^{\circ} \mathrm{C}$. This strong shift in melting temperature shows that the disulphides are indeed important to maintain the structure of VDE. The increased radius of reduced VDE also shows that the disulphides keep flexible domains in position. Size-exclusion chromatography of reduced and non-reduced VDE gives similar results, where reduced VDE elutes before non-reduced VDE (data not shown).

\section{Discussion}

The results show that all cysteines in VDE, except the first one, are important for the enzymatic activity, form disulphides and do not seem to be involved in metal binding. Five out of six possible disulphide bonds were found using mass spectroscopy. A sixth disulphide bond is predicted to connect Cys 9 and Cys 27 since the quantification of free cysteines suggests only one free cysteine per VDE molecule for the native sequence and none for the C7S mutant, which means that Cys 7 should be the free, unbound cysteine. There is also the possibility that Cys 9 and Cys 27 have been chemically modified, therefore not reacting as thiols.

The disulphide pattern, obtained by mass spectroscopy, is not similar to the disulphide bonds suggested by disulphide prediction tools. DISULFIND (Ceroni et al. 2006) predicts no disulphides that match the disulphide pattern from our mass spectroscopy data. DiANNA (Ferrè and Clote 2005) gives only one disulphide bond with a probability score above 0.9 and agrees with the disulphide pattern in Fig. 2, which is the Cys 9-Cys 27 predicted disulphide. The weighted matching also predicts the Cys 14-Cys 21 disulphide, which our experimental data can support. The disulphide between Cys 118 and Cys 248 located inside the lipocalin domain, confirmed by the structure of VDE-lipocalin from A. thaliana, could not be predicted by either DISULFIND or DiANNA. That these prediction tools cannot match the correct disulphides could be an effect of the high number of cysteines, which the tools can not handle or that this disulphide pattern are very different from the ones used as basis for comparison in the prediction programs.

When comparing the disulphide pattern with the activity of the cysteine mutants, the activity for one disulphideforming cysteine mutant was often similar to the activity of 


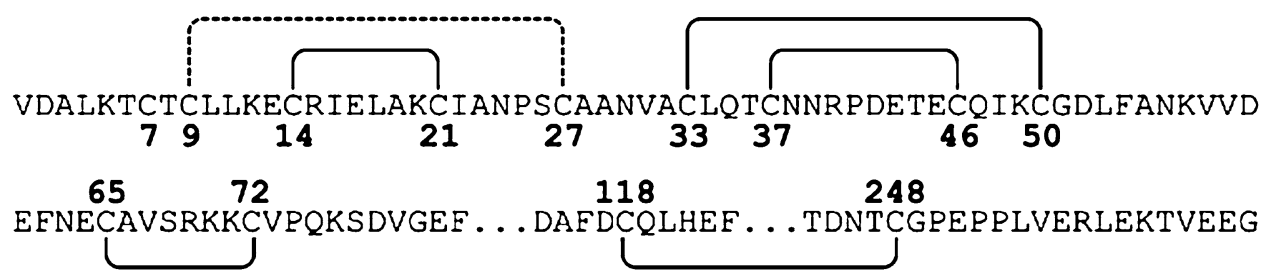

Fig. 2 The disulphide bond pattern of spinach VDE. The solid lines represent disulphide bonds confirmed with mass spectroscopy data. The dashed line is the disulphide bond predicted by thiol quantification

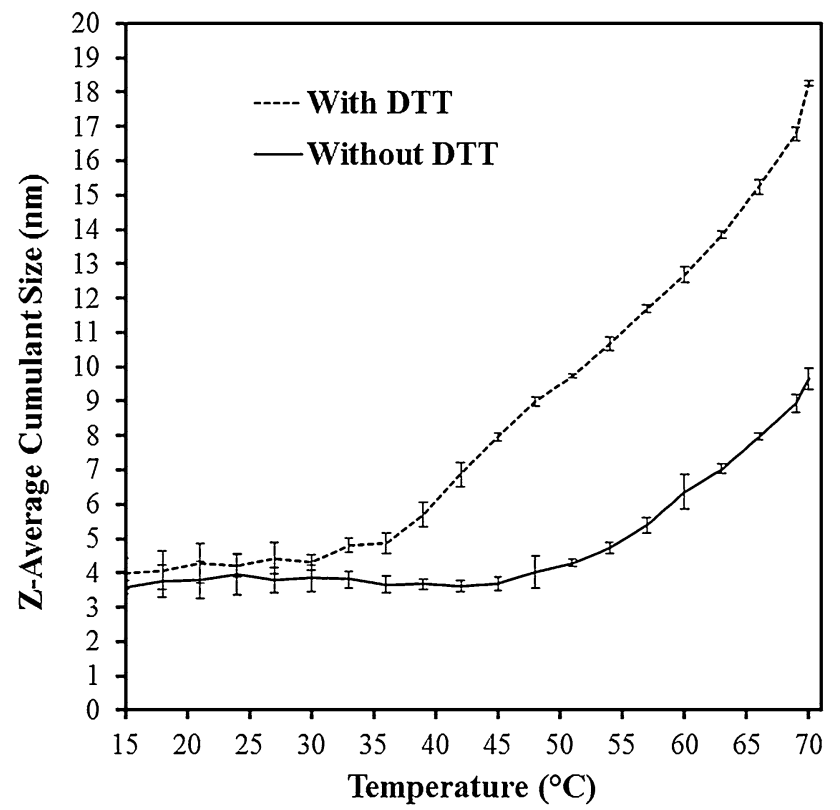

Fig. 3 Temperature denaturation of spinach VDE in reduced (dashed line) and in non-reduced (solid line) form. VDE, reduced with DTT appears larger than non-reduced VDE and shows a decrease in melting temperature of $15^{\circ} \mathrm{C}$

the mutant with a mutation of the corresponding disulphide-forming cysteine. If the loss of activity was caused only by the loss of a disulphide bond, the mutation of any of the two disulphide-forming cysteines would result in the same amount of activity loss. Therefore, the similar activity loss from a mutation of a cysteine, forming a disulphide bond, and a mutation of the other cysteine in this disulphide bond is an indication that it is the loss of the disulphide bond that causes the loss of activity.

The disulphide pattern obtained here is also expected to be present inside the plant cells in vivo despite that the inside of living cells generally has reducing conditions. The arguments for this is that the in vitro form of VDE needs all six disulphides to show full enzyme activity, and that VDE whether studied in vitro or within the leaf is inhibited by DTT. One possibility is that in the thylakoid lumen in the dark, the cysteines in VDE are in the reduced form. Upon illumination of leaves, photosynthesis starts to produce oxygen and makes the lumen more oxidising, which would allow disulphides to be formed. However, artificial acidification of whole leaves without illumination induces conversion of violaxanthin to zeaxanthin (Clausén et al. 2010). Thus, VDE can be active in the leaves in the dark and the disulphides should therefore be present also in the dark. The question then still remains how the disulphides in VDE are originally formed. The condition inside the thylakoid lumen may be less reducing than the cell as a whole to allow the formation of all the disulphide bonds required for the activity of VDE. That the increased conversion of violaxanthin to zeaxanthin caused by various stress treatment (Fernández-Marín et al. 2011) could be an effect of stronger oxidative conditions, which would favour the formation of disulphide bonds and an activation of VDE. The reactivation of reduced VDE is possible in vitro after the removal of reducing agent, as demonstrated by the procedure used in this work to make active VDE, showing that the correct disulphide bonds form spontaneously. In vivo this process could, however, also be catalysed by other proteins. Thioredoxin have been shown in vitro to interact and inactivate VDE (Hall et al. 2010). However, thioredoxin has not been found in the thylakoid lumen, but the lumen thiol oxidoreductase (LTO1/AtVKOR) may possibly fulfil this function. LTO1/AtVKOR is located in the thylakoid membrane with a thioredoxin-like domain oriented to the thylakoid lumen (Karamoko et al. 2011). Recent results from studies on an lto1-2 mutant, lacking functional LTO1 (Lu et al. 2013), and a yeast two-hybrid assay (Lu et al. 2014) show that both functional and physical interaction between LTO1 and VDE is possible.

The reaction catalysed by VDE is unusual, but the enzyme vitamin $\mathrm{K}$ epoxide reductase (VKOR) also catalyses de-epoxidation. In this case, vitamin $\mathrm{K}$ 2,3-epoxide is converted to vitamin $\mathrm{K}$, in the blood coagulation process. This enzyme is suggested to utilise a disulphide bond that first is reduced, and then oxidised, to remove the epoxide and form a double bond (Silverman 1981; Davis et al. 2007). The possibility that a disulphide in VDE may be involved directly in the enzymatic reaction is therefore tempting. Considering that the conversion of violaxanthin to antheraxanthin is a de-epoxidation reaction to form a double bond and that VDE has disulphides that are important for this enzymatic reaction, a mechanism similar to 
the one in VKOR might be possible. The initial reduction of the disulphide in this mechanism might then be caused by ascorbate. However, more experimental evidences are needed to establish such a mechanism. An alternative to this mechanism is a concerted reaction without the involvement of disulphides, and that the disulphides only maintain a rigid structure of VDE. The loss of a more rigid structure is what is shown in Fig. 3, where reduced VDE appears to be larger than non-reduced VDE. A less rigid structure could be a signal for degradation of VDE, as for the case of PsbO, which after reduction of a disulphide bond becomes sensitive to proteolysis (Kieselbach 2013). This rigid structure could also be important for the protein stability in an environment that can undergo large shifts in $\mathrm{pH}$, such as the thylakoid lumen, where the $\mathrm{pH}$ can vary from 5.7 to 7.5 depending on light conditions (Takizawa et al. 2007).

In conclusion, we have mutated the cysteines of spinach VDE one by one and observed major activity loss for 12 out of 13 mutants. Using mass spectroscopy five out of six possible disulphide bonds could be identified, while the sixth disulphide was indirectly identified. Reduction of these disulphides opens up the structure of VDE and decreases the thermal stability by $15^{\circ} \mathrm{C}$.

Acknowledgments This work was supported by Carl Trygger Foundation.

Open Access This article is distributed under the terms of the Creative Commons Attribution License which permits any use, distribution, and reproduction in any medium, provided the original author(s) and the source are credited.

\section{References}

Arnoux P, Morosinotto T, Saga G, Bassi R, Pignol D (2009) A structural basis for the $\mathrm{pH}$-dependent xanthophyll cycle in Arabidopsis thaliana. Plant Cell 21:2036-2044. doi:10.1105/tpc. 109.068007

Arvidsson PO, Bratt C, Carlsson M, Åkerlund HE (1996) Purification and identification of the violaxanthin deepoxidase as a $43 \mathrm{kDa}$ protein. Photosynth Res 49:119-129. doi:10.1007/BF00117662

Bradford M (1976) A rapid and sensitive method for the quantitation of microgram quantities of protein utilizing the principle of protein-dye binding. Anal Biochem 72:248-254. doi:10.1016/ 0003-2697(76)90527-3

Ceroni A, Passerini A, Vullo A, Frasconi P (2006) DISULFIND: a disulfide bonding state and cysteine connectivity prediction server. Nucleic Acids Res 34(2):W177-W181. doi:10.1093/nar/ gkl266

Clausén M, Huang S, Emek S, Sjöholm I, Åkerlund HE (2010) Post harvest improvement of zeaxanthin content of vegetables. J Food Eng 98(2):192-197. doi:10.1016/j.jfoodeng.2009.12.025

Davis C, Deerfield D, Wymore T, Stafford D, Pedersen L (2007) A quantum chemical study of the mechanism of action of Vitamin K epoxide reductase (VKOR) II. Transition states. J Mol Graph Model 26(2):401-408. doi:10.1016/j.jmgm.2006.10.005
Demmig-Adams B (1990) Carotenoids and photoprotection in plants: a role for the xanthophyll zeaxanthin. Biochim Biophys Acta 1020(1):1-24. doi:10.1016/0005-2728(90)90088-L

Emanuelsson A, Eskling M, Åkerlund HE (2003) Chemical and mutational modification of histidines in violaxanthin de-epoxidase from Spinacia oleracea. Physiol Plant 119:97-104. doi:10.1034/j. 1399-3054.2003.00151.x

Eskling M, Arvidsson P-O, Åkerlund HE (1997) The xanthophyll cycle, its regulation and components. Physiol Plant 100:806-816. doi:10.1034/j.1399-3054.1997.1000407.x

Fernández-Marín B, Míguez F, Becerril J, García-Plazaola J (2011) Activation of violaxanthin cycle in darkness is a common response to different abiotic stresses: a case study in Pelvetia canaliculata. BMC Plant Biol 11:181. doi:10.1186/1471-222911-181

Ferrè F, Clote P (2005) DiANNA: a web server for disulfide connectivity prediction. Nucleic Acids Res 33(2):W230-W232. doi:10.1093/nar/gki412

Hall M, Mata-Cabana A, Åkerlund HE, Florencio F, Schröder W, Lindahl M, Kieselbach T (2010) Thioredoxin targets of the plant chloroplast lumen and their implications for plastid function. Proteomics 10:987-1001. doi:10.1002/pmic.200900654

Hieber D, Bugos R, Verhoeven A, Yamamoto H (2002) Overexpression of violaxanthin de-epoxidase: properties of C-terminal deletions on activity and $\mathrm{pH}$-dependent lipid binding. Planta 214(3):476-483. doi:10.1007/s00425-001-0704-2

Ho S, Hunt H, Horton R, Pullen J, Pease L (1989) Site-directed mutagenesis by overlap extension using the polymerase chain reaction. Gene 77:51-59. doi:10.1016/0378-1119(89)90358-2

International Organisation for Standardisation (1996) International standard ISO13321 methods for determination of particle size distribution part 8: photon correlation spectroscopy, International Standard ISO 13321. ISO, Geneva

Karamoko M, Cline S, Redding K, Ruiz N, Hamel PP (2011) Lumen thiol oxidoreductase1, a disulfide bond-forming catalyst, is required for the assembly of photosystem II in Arabidopsis. Plant Cell 23(12):4462-4475. doi:10.1105/tpc.111.089680

Kieselbach T (2013) Oxidative folding in chloroplasts. Antioxid Redox Signal 19(1):72-82. doi:10.1089/ars.2012.4582

Kramer EB, Farabaugh PJ (2007) The frequency of translational misreading errors in E. coli is largely determined by tRNA competition. RNA 13:87-96. doi:10.1261/rna.294907

Lu Y, Wang HR, Li H, Cui HR, Feng YG, Wang XY (2013) A chloroplast membrane protein LTO1/AtVKOR involving in redox regulation and ROS homeostasis. Plant Cell Rep 32(9):1427-1440. doi:10.1007/s00299-013-1455-9

Lu Y, Du JJ, Yu ZB, Peng JJ, Xu JN, Wang XY (2014) Identification of potential targets for thylakoid oxidoreductase AtVKOR/LTO1 in chloroplasts. Protein Pept Lett 22(3):219-225. doi:10.2174/ 0929866521666141121153138

Niyogi KK, Grossman AR, Björkman O (1998) Arabidopsis mutants define a central role for the xanthophyll cycle in the regulation of photosynthetic energy conversion. Plant Cell 10(7):1121-1134. doi:10.1105/tpc.10.7.1121

Saga G, Giorgetti A, Christian Fufezan C, Giorgio M, Giacometti G, Bassi R, Morosinotto T (2010) Mutation analysis of violaxanthin de-epoxidase identifies substrate-binding sites and residues involved in catalysis. J Biol Chem 285:23763-23770. doi:10. 1074/jbc.M110.115097

Sedlak J, Lindsay R (1968) Estimation of total, protein-bound, and nonprotein sulfhydryl groups in tissue with Ellman's reagent. Anal Biochem 25(1):192-205. doi:10.1016/0003-2697(68)90 092-4

Silverman R (1981) Chemical model studies for the mechanism of vitamin K epoxide reductase. J Am Chem Soc 103(19):5939-5941. doi:10.1021/ja00409a068 
Takizawa K, Cruz JA, Kanazawa A, Kramer DM (2007) The thylakoid proton motive force in vivo. Quantitative, non-invasive probes, energetics, and regulatory consequences of light-induced pmf. Biochim Biophys Acta 1767:1233-1244. doi:10.1016/j. bbabio.2007.07.006

Thayer S, Björkman O (1990) Leaf Xanthophyll content and composition in sun and shade determined by HPLC. Photosynth Res 23:331-343. doi:10.1007/BF00034864
Yamamoto HY, Higashi RM (1978) Violaxanthin de-epoxidase: lipid composition and substrate specificity. Arch Biochem Biophys 190:514-522. doi:10.1016/0003-9861(78)90305-3

Yamamoto HY, Kamite L (1972) The effects of dithiothreitol on violaxanthin de-epoxidation and absorbance changes in the $500 \mathrm{~nm}$ region. Biochim Biophys Acta 267:538-543. doi:10. 1016/0005-2728(72)90182-X 